$$
\text { "tonisson" — 2008/9/1 — 18:16 — page } 125 \text { — \#1 }
$$

\title{
Answers offered by computer algebra systems to equations from school textbooks
}

\author{
Eno Tonisson and Nadezhda Velikanova
}

\begin{abstract}
This paper is an attempt to develop a strategy and methods for investigating and comparing the answers offered by computer algebra systems and the school answers. After primary (pilot) investigation of how well 8 computer algebra systems handle equations from school textbooks, it is possible to conclude that the systems are mostly reliable and give reasonable answers. Some remarks regarding a somewhat unexpected answer obtained can be easily explained by built-in standards and notions, which can differ from school assumptions. In other cases the differences from school could be corrected by certain commands.
\end{abstract}

Key words and phrases: computer algebra systems, school algebra, equations.

ZDM Subject Classification: U53, U54, R23, R24, N83, N84, H33, H34.

\section{Introduction}

Computer algebra systems (CASs) are powerful programs for mathematics (algebra, trigonometry, calculus, etc); they perform mainly symbolic but also numerical computations. CASs were initially designed mainly to help professional users of mathematics, and were not directly intended for use in mathematics education. Therefore, they may have different notions and standards than those used in schools. This situation is currently improving and more systems are created or modified taking into consideration the school problems and needs, but there are still some differences. It is therefore important to investigate how different computer algebra systems could be most effectively used at school.

Copyright (C) 2008 by University of Debrecen 
Many articles and books discuss how computer algebra systems could be used in teaching mathematics (e.g., [4]). Different researches analyze CASs from different points of view, based on various experiments, for example, how the working style of the students changes when using CASs (e.g., [10]). It is very useful, but teachers also need to know more about the exact behavior of CASs in case of ordinary school problems. Therefore, various tests should be conducted in order to find out how well one or another CAS handles school problems.

An extensive research in the comparison of CASs was conducted by Michael Wester ([11]). He compared how 7 different computer algebra systems (Axiom, Derive, Macsyma, Maple, Mathematica, MuPAD, Reduce) deal with a chosen set of problems. All the results are collected into one table showing the overall tendencies, shortages and patterns. A total of 542 problems were solved. Approximately 100 of them are similar to those usually taught at schools in regular or advanced math classes. Less than half of school-related problems were solved more or less correctly (according to Michael Wester, it is estimated that 5 or more programs give correct or nearly correct answer).

The differences between the answers of CASs themselves and the school answers, as well as the fact that there are not enough studies that focus on school exercises encourage further study in this area. This paper is an attempt to develop a strategy and methods for investigating and comparing the answers offered by CASs and the school answers. As it is a laborious task, we have set some boundaries. This study that focuses on solving the equations with the help of eight CASs is important as an independent research but also as an attempt to apply the developed strategy. In this paper we concentrate on the immediate one-step solving (student enters the equation and program gives the answer). Solving a problem in one step is the basic use of CASs. In this case the student does not have to think about the order of the steps in the correct solution and, therefore, can concentrate more on the other important aspects like interpreting the results, reasoning or predicting the answer. The didactic basis for "white box / black box" principles can be found in the article [3].

CASs can do both symbolic and numerical calculations and both these options are used in the research but symbolic computation is somewhat preferred. We used usual commands for equation solving, mainly the command Solve. In this paper we investigate computer algebra systems Derive, Maple, Mathcad, Mathematica, MuPAD, TI-92 Plus, TI- $n$ spire CAS and WIRIS. It is important to note that CASs are continuously changing from version to version. The versions used 


$$
\text { "tonisson" — 2008/9/1 — 18:16 — page } 127 \text { — \#3 }
$$

in test are listed in the beginning of Section 3. Other versions may give different answers and not produce such unexpected results as described in this article. In that case the analysis should be modified. Examples of the equations are taken from Estonian textbooks but they are quite similar to the ones taught in other countries.

Analysis is based on the obtained roots, the unexpected results offered by computer algebra programs and other shortages. Such analysis would help to decide, how well CASs are suited for using at school, because even very powerful CASs with extensive functionality can produce answers that are insufficient at school. It is also interesting to know if systems work similarly for different types of equations and give the same answers. It is important to emphasize that the aim of this paper is not a criticism of any particular CAS but rather description of a variety of approaches.

Section 2 gives an overview of the classification that is used for the investigation of CASs and corresponding examples. One of the goals is to check, whether the classification of school equations is suitable for investigating computer algebra systems. The third section presents the description of the types of the answers offered by the CASs. The results are classified under four types and some advice is given for teachers about how they should behave when encountering a certain type. There are some possible actions of teachers briefly discussed in Section 4 . The paper based on [9].

\section{School mathematic equations: classification and examples}

Investigation of CASs requires certain classification of equations taught at school in order to be able to test the instances of each type of equations. Looking through school textbooks, one may note that the (explicit) classification is somewhat poor and not enough detailed for making valuable and valid conclusions. For more thorough tests another, more detailed, classification is required. We also searched the Internet and other available literature to find better classification, but it seems that there is none. The classification described below is simple, but it gives us more detailed overview about the types of equations that are usually taught at school and allows us to investigate the computer algebra systems more carefully, checking a pair of representatives from each smaller subtype (one simpler and one more complicated equation). There are mixed equations that could not be easily classified under a certain type. For example, if their structure 
includes roots and signs of absolute values, then they are irrational equations containing absolute value and can not be classified under a specific subtype, because their solving methods tend to differ greatly from each other (solving methods, algorithms). These mixed types of equations are rarely solved during class because of the lack of time and their complexity. Taking that into consideration, these more complicated equations are at first almost left out of the research. However, there are several simpler mixed equations used in this paper. As they could be classified under different subtypes, they are assumed to belong to the subtype that is taught later in the curriculum. For instance, if an equation includes roots and powers, it is assumed that it belongs to the exponential equations, as exponential equations are taught later at school than irrational ones.

The obtained classification is based only on school mathematics; the possibilities of CASs were not taken into consideration. For the research several Estonian and Russian textbooks were analyzed. It is likely that textbooks from other countries are similar. Looking through textbooks and the national curriculum of Estonia, eight most important types of equations were identified. These types are: linear, quadratic, fractional, equations that contain absolute value of an expression, irrational, exponential, logarithmic and trigonometric equations. Every general type of equations was in turn divided into several subtypes (35 subtypes in total). The subtypes are

- in case of linear equations:

In the form of $a x+b=0$;

In the form of $a x=0$;

Pseudolinear equation in the form $b=0$;

- in case of quadratic equations:

In the form of $a x^{2}+c=0$;

In the form of $a x^{2}+b x=0$;

In the form of $a x^{2}+b x+c=0$ with 2 real roots, $b^{2}-4 a c>0$;

In the form of $a x^{2}+b x+c=0$ with 1 double root, $b^{2}-4 a c=0$;

In the form of $a x^{2}+b x+c=0$ with no real roots, $b^{2}-4 a c<0$;

Pseudoquadratic equation;

- in case of fractional equations:

One fractional expression and zero;

Two fractional expression or one fractional expression and a number;

Three or more fractional expressions or two fractional expressions and a number; 


$$
\text { "tonisson" — 2008/9/1 — 18:16 — page } 129-\# 5
$$

- in case of equations that contain absolute value of an expression: One absolute value of the expression and number;

Only two absolute values of the expressions;

Other equations containing the absolute value and numbers;

- in case of irrational equations:

Solved by involution;

Reducible to the quadratic;

Solved by using method of substitution;

Other irrational equations;

- in case of exponential equations:

Equations in the form of $a^{x}=b$;

Solved by using properties of the power and factorizing;

Reducible to the linear;

Reducible to the quadratic;

Homogeneous equation of second power;

- in case of logarithmic equations:

Equation in the form $\log _{a} x=b$;

More difficult equation that is reducible to the form $\log _{a} x=b$;

Reducible to quadratic;

Exponential equation solved by taking the logarithm;

- in case of trigonometric equations:

General equation in the form of $\sin x=a, \cos x=a, \tan x=a, \cot x=a$;

Reducible to the quadratic ( $\sin , \cos$ );

Reducible to the quadratic (incl. tan, cot);

In the form $a \sin x+b \cos x=0$;

In the form $a \sin x+b \cos x=k$;

Homogeneous equation of second power;

Other trigonometric equations.

This classification is not complete and can be improved. It can be considered as a brief sketch for approximate delimitation and listing of the problems. Defining more specific boundaries would require much more thorough experiments and analysis. In this paper some equations are essentially disregarded (complicated mixed equations) and some equations can belong to two subtypes (for example, pseudoquadratic equations can be transformed after some operations to linear form and, therefore, can be classified as linear equations). Nevertheless, even such partial classification facilitates investigation of computer algebra systems. 


$$
\text { "tonisson" — 2008/9/1 — 18:16 — page } 130 \text { — \#6 }
$$

Teachers usually do the same work by dividing more general types of equations into subtypes in their lessons in order to make understanding of specific topics easier for students. Consequently, this classification should be close and understandable to teachers. It is likely that such classification is common for all schools in the world; the teaching may only differ in the amount of solved equations and their difficulty. Some schools may be more thorough in certain topics that are considered to be more important. Still, the main list of equations solved at school remains relatively unchanged.

The aim in selecting test examples was to find a pair of representatives for each subtype. One equation was chosen from the beginning of each textbook chapter that deals with the corresponding topic in order to show the most typical representative of the subtype of equations. The second, more complicated, problem was chosen from the end of the chapter or even from advanced lesson manuals.

If solving a certain type of equation was very easy and all equations belonging to this type were very similar then only one representative was chosen (in case of linear equations, for example). More than two problems were chosen from certain subtypes (e.g., irrational equations) to ensure that most of the possible cases within one subtype would be covered if there was no need to break it into separate subtypes. A total of 60 equations were solved. To save space, we explain here as an example only one subtype of fractional equation. In case of the subtype One fractional expression and zero the examples are

$$
\frac{x+1}{x-1}=0 \quad \text { and } \quad \frac{2 x^{2}-5 x+3}{10 x-2}=0 .
$$

\section{Analysis of the answers}

We used eight computer algebra systems (Derive 6 (Drv in table), Maple 8 (Mpl), MathCAD 2001 (Mcad), Mathematica 5.2 (Mmca), MuPAD 3.1 (MuP), TI-92+ (TI), TI- $n$ spire (TI), WIRIS (WRS)) in our tests. The command Solve was used for the most part. However, some other commands (for example, fsolve) were tried as well. It is should be noted that other CASs and other versions of current CASs could work differently. After solving all 60 equations (see previous section), some results were noticed that could be somewhat unexpected from the school point of view. Some were common to the majority of tested programs while others appeared only in single cases. It is noteworthy that the expectations may 
be also different in case of different countries or even different teachers. Obtained results can be grouped in several ways based on different dimensions and criteria. In this paper we use a classification that is simpler (and hopefully more useful for teachers) than the one proposed, for instance, in the article of David Stoutemyer, where the limitations are divided into four groups: bugs, theoretical, algorithmic and resource limitations [5].

We pose two questions for every answer: Is the answer equivalent to the answer required at school? and Could the answer be easily transformed into the required form? It is quite complicated to answer yes/no in some cases. However, we distinguish four types listed in the Table 1 . It is necessary to note that transforming in our situation keeps equivalence (or non-equivalence), for example, command Simplify is used. In the following text we discuss only types 1, 2 and 3 ; the correct answers in suitable form are not discussed separately.

Table 1. Types of obtained answers

\begin{tabular}{|c|l|c|l|}
\hline No & Type & Equivalent? & Easily transformable? \\
\hline 1 & $\begin{array}{l}\text { Answer is not equivalent to the } \\
\text { answer required at school }\end{array}$ & no & $\begin{array}{l}\text { yes/no, anyway keeps } \\
\text { non-equivalence }\end{array}$ \\
\hline 2 & $\begin{array}{l}\text { Answer is equivalent but could } \\
\text { not be easily transformed into } \\
\text { the required form }\end{array}$ & yes & no \\
\hline 3 & $\begin{array}{l}\text { Answer is equivalent and is easily } \\
\text { transformed into the required } \\
\text { form }\end{array}$ & yes & yes \\
\hline OK & Correct answer in suitable form & yes & not needed \\
\hline
\end{tabular}

If there are remarks that belong to the second or third type, then we may say that a particular CAS handles a certain type of equations quite well as such remarks do not influence (at least too much) the quality of solving the equations. A teacher should be more concerned if the result belongs to the first type (answer is not equivalent to the answer required at school).

Let us look at some examples from each type. One reason for the first type Answer is not equivalent to the answer required at school may be a bug in the program. Such errors are rare in modern CASs. More often, non-equivalence to school answer can be explained by the standards of particular CAS that differ from school notions. For example, Derive sometimes gives infinity in the answer, 
e.g., equation

$$
\frac{x}{x-2}-\frac{3}{x}=1
$$

has roots $x= \pm \infty$ and $x=6$ in Derive. However, taking in consideration the standards of Derive (where division by the infinity is equal to zero) such answer is also acceptable.

In case of equation

$$
x^{\sqrt{x}}=(\sqrt{x})^{x},
$$

Derive and MuPAD find $3 \operatorname{roots} x=4 \vee x=1 \vee x=0$. Checking the root $x=0$ gives expression $0^{0}$, which is declared to be undefined. Therefore, the student at school obtains only two roots $\left(x_{1}=1\right.$ and $\left.x_{2}=4\right)$. At the same time, the programs Derive and MuPAD have the assumption that $0^{0}=1$ and for Derive and $\mathrm{MuPAD}$ the root $x=0$ really suits given equation.

The topic of infinities and indeterminates is discussed in [2], [8].

The next example of the first type is the case when instead of two correct roots CAS gives only one, while solving at school the student obtains 2 roots. A student solving the irrational equation

$$
\sqrt[3]{x+45}-\sqrt[3]{x-16}=1
$$

on paper would get 2 roots $\left(x_{1}=-109\right.$ and $\left.x_{2}=80\right)$, but the programs Mathcad, MuPAD and Mathematica give only one (positive) root $x=80$, ignoring the possibility of negative number under the sign of root with odd index. It is notable that the result could depend on the form of expression. For example, in Maple using $\operatorname{expr}^{\wedge}(1 / 3)$ and $\operatorname{surd}(\operatorname{expr}, 3)$ give different answers. A graphical analysis of an equation could be useful.

Trigonometric equations may be inconvenient to teach with the help of computer algebra systems, because only three programs out of eight (MuPAD, TI92 Plus and TI- $n$ spire) take (partially) into consideration the periodicity of the trigonometric functions (in case of simple commands!) and only MuPAD describes the variable $n$ in the answer $(n \in \mathbb{Z})$. Others systems only calculate some particular solutions and give them as an answer, which would be insufficient at school. It is noteworthy that Mathematica, TI-92+ and TI- $n$ spire add warning messages when presenting particular solutions, e.g., Some solutions will be lost or Some solutions may not be found, but this information is not particularly useful for a student. The system may have some possibilities for more thorough answers. For example, if in Maple one sets the environmental variable _EnvAllSolutions to true then the answer is complete. 


$$
\text { "tonisson" — 2008/9/1 — 18:16 — page } 133 \text { — \#9 }
$$

One way to overcome this difficulty is to solve trigonometric equations graphically (plot the graph of given trigonometric function and from the graph read roots). After analyzing the obtained results, we may conclude that the school handles trigonometric equations more precisely from the mathematical point of view than the majority of the investigated CASs, because only MuPAD almost always gives the right general answer.

All studied computer algebra systems may write answers somewhat differently than they are written at school. In many cases such differences could not be easily corrected in CASs by the users and we classify these cases as type "2" (answer is equivalent but can not be easily transformed to the required form). As such differences could not be easily eliminated by the students, the teacher simply has to notify the students beforehand about such differences and give possible explanations. For example, sign $\vee(\mathrm{OR})$ is used in Derive when there is more than one answer to indicate, that the unknown variable can take one or another value (for instance, $x=1 \vee x=2$ ). However, in younger classes the students are not familiar with this logical sign and teachers have to explain this sign before getting to work with Derive.

Some questions are related to the fractions. For example, is it suitable for the final answer when the minus sign is in the numerator? Or should $\frac{11}{5}$ be transformed further? Teacher may expect $2 \frac{1}{5}$ or 2.2 . Some of such transformations are not easily possible in particular CAS and the answer is classified under type 2. If the transformation is easy to perform in a particular CAS, the type is 3 .

A classic example of the third type (equivalent answer that is easily transformed into the required form) is solving equation $4^{x}=64$ in Maple. The answer is $\frac{\ln (64)}{\ln (4)}$ and the student would have to use some additional commands in order to get result that would be suitable. In this case, the command Simplify transforms the answer to 3. It is improved in the new versions of Maple - Maple 10 gives the correct answer directly.

Sometimes it is quite difficulty to make classifications. For example, some CASs are even more precise than would be needed at school, namely, in case of the quadratic equations with two equal roots $\left(x^{2}-2 x+1=0\right)$, Maple, Mathcad and Mathematica give the root 1 twice, while textbooks may say that equation has one (double) root. In addition, different textbooks have different opinions in this matter. Some say that equation has only 1 root [12] or since it came from two factories some can call it double root [1]. In this paper we consider both variants correct. (See also [6].) The obtained results show that computer algebra systems deal with the quadratic equations practically in the same way as the school, but 
sometimes answers proposed by CASs are more precise than the ones proposed at school.

Table 2. Exists answer in particular type

\begin{tabular}{|l|c|c|c|c|c|c|c|}
\hline & Drv & Mpl & Mcad & Mmca & MuP & TI & WRS \\
\hline Linear & 2,3 & 2,3 & 3 & 2,3 & 2,3 & 2,3 & 2,3 \\
\hline Quadratic & 2,3 & 2,3 & 2,3 & 2,3 & 2,3 & 2,3 & 2,3 \\
\hline Fractional & $1,2,3$ & 2,3 & 2,3 & 2,3 & 2,3 & 3 & 2,3 \\
\hline $\begin{array}{l}\text { Containing } \\
\text { absolute value }\end{array}$ & 2,3 & 2 & 2 & 2 & 1,2 & 3 & 2 \\
\hline Irrational & 1,2 & 1,2 & 1,2 & 1,2 & 1,2 & 2 & 2 \\
\hline Exponential & 2,3 & 3 & 3 & 1,2 & 1,2 & 2 & 2 \\
\hline Logarithmic & 1,2 & 1 & 2 & 2 & 1,2 & 2 & 2 \\
\hline Trigonometric & 1,2 & 1 & 1,2 & 1,2 & 1,2 & 1,2 & 1,2 \\
\hline
\end{tabular}

Table 2 presents what types are found in case of different CASs and equations. The number in table indicates that there is at least one answer in particular type. It means that other answers may be (and in many cases are) suitable for the school. As the answers of TI- $n$ spire and TI-92+ differ only very slightly (order of solutions), they are presented in one column.

\section{Unexpected answers and some possible actions}

It can be seen from the previous section that CASs can handle school equations well. Some inaccuracies were noticed only in the case of more complicated examples. Other remarks relate mostly to the form of the answer, but it is not a major problem as there is no common standard for the exact form of the answer. Should the fraction be reduced? Is it necessary to order the roots increasingly or not? Different teachers, schools and countries have different requirements for the answers. Such common remarks can be easily explained or changed with the help of some additional steps that do not influence the quality of teaching and learning. Thorough practical experiments at school should be made and feedback from teachers should be gathered and analyzed afterwards to give more useful and detailed advice. 


$$
\text { "tonisson" — 2008/9/1 - 18:16 — page } 135 \text { — \#11 }
$$

Table 3. Shortages and possible actions of the teacher

\begin{tabular}{|c|c|c|c|}
\hline No & Type & Possible actions & Command \\
\hline 1 & $\begin{array}{l}\text { Answer is not } \\
\text { equivalent to the } \\
\text { answer required at } \\
\text { school }\end{array}$ & $\begin{array}{l}\text { Solve equations and explain } \\
\text { the reason of the error } \\
\text { or } \\
\text { Do not use equations that } \\
\text { can produce errors }\end{array}$ & $\begin{array}{l}\text { Commands for } \\
\text { solving equations }\end{array}$ \\
\hline 2 & $\begin{array}{l}\text { Answer is equivalent } \\
\text { but can not be } \\
\text { easily transformed } \\
\text { to the required form }\end{array}$ & $\begin{array}{l}\text { Solve equations and explain } \\
\text { the record } \\
\text { or } \\
\text { Do not use equations that } \\
\text { can produce such situations }\end{array}$ & $\begin{array}{l}\text { Commands for } \\
\text { solving equations }\end{array}$ \\
\hline 3 & $\begin{array}{l}\text { Answer is } \\
\text { equivalent and is } \\
\text { easily transformed } \\
\text { to the required form }\end{array}$ & $\begin{array}{l}\text { Solve equations and explain } \\
\text { how to change the form to } \\
\text { the required one }\end{array}$ & $\begin{array}{l}\text { Commands for } \\
\text { solving equations } \\
\text { and simplifying the } \\
\text { expression }\end{array}$ \\
\hline
\end{tabular}

For instance, one field that presents different opportunities is trigonometry. Namely, only three programs, MuPAD and partially TI-92 Plus and TI- $n$ spire, give the general solution that takes into consideration the periodicity of trigonometric functions. The others calculate some particular solutions (at least with usual commands). This disadvantage can be used by teachers. For example, the teachers can ask students to find which values of the parameter $n$ from the general answer correspond to certain answers proposed by the CAS. This exercise helps the students to understand the general answer better and learn to think abstractly. Table 3 gives very brief advice on what a teacher could do if he or she had noticed an answer of a type described in previous Section. (Several issues were already mentioned in previous Section.)

\section{Conclusion and future work}

The main aim of this paper was to develop a strategy for researching and comparing CASs, which could be then used to investigate how well eight computer algebra systems handle equations from school textbooks. The strategy consists of the following phases: 


$$
\text { "tonisson" — 2008/9/1 — 18:16 — page } 136 \text { — \#12 }
$$

- choice of mathematical field

- classification of exercises, choice of test examples of exercises

- posing criteria for the categorization of CAS answers

- choice of CASs and commands

- analysis of the results, categorizing of answers

- analysis of dependencies, patterns, etc.

The strategy generally seems to be suitable. However, the strategy could be improved by revisiting the classification of school exercises after the tests with CASs. Our approach was to classify equations based only on school mathematics and the possibilities of CASs were not taken into consideration. Actually, in this case it is quite possible that some interesting phenomena may remain uncovered (particularly, because there were only two test examples from each subtype). (Some of such issues are discussed in [7].)

Nevertheless, our classification may be sufficiently detailed for primary investigation but more detailed classification would be required for more thorough research.

The choice of CASs depends on accessibility and aims. A teacher usually has one (or a few) CAS and it is the only one that needs testing in this case. The choice of commands is very important. The usual command Solve with default settings is only one possibility. In reality, the proper use of different commands, assumptions and settings could make a CAS more suitable for school mathematics. For example, there are special tools for determination of real or complex domain. A wide variety of means in different CASs should be the object of further study.

The categorization of CAS answers based on two questions: Is the answer equivalent to the answer required at school? and Is the answer easily transformed into the required form? is promising but not very trivial task. The main problem is the wide range of requirements for answers in case of different countries, schools and teachers. Other possibilities of categorization are naturally possible and may be more reasonable. After primary (pilot) investigation of how well eight computer algebra systems handle equations from school textbooks, it is possible to conclude that the systems are mostly reliable and give reasonable answers. As this paper underlines mainly cases that are somewhat problematical, it could create a deceptive impression that there are major problems. Some remarks regarding the obtained answer can be easily explained by built-in standards and notions, which can differ from school assumptions. In other cases the differences from school could be corrected by some commands. The eight programs investigated (Derive, 


$$
\text { "tonisson" — 2008/9/1 — 18:16 — page } 137 \text { — \#13 }
$$

Maple, Mathcad, Mathematica, MuPAD, TI-92 Plus, TI- $n$ spire CAS and WIRIS) work similarly for tested equations and give essentially the same results.

More questions have arisen during this study concerning irrational, logarithmic, trigonometric equations and these questions should be explored. It is possible to look at this area from a slightly different point of view, for example, study separately the questions of real and complex domain, branching (see [6]) and equivalence. Hopefully, it will help the students and teachers use CASs more fruitfully.

\section{Acknowledgement}

This research is supported by the Estonian Science Foundation (project ETF7180) and the Estonian Information Technology Foundation.

\section{References}

[1] R. A. Barnett, M. R. Ziegler, College Algebra, McGraw-Hill, 1989.

[2] M. Beeson, F. Wiedijk, The meaning of infinity in calculus and computer algebra systems, Journal of Symbolic Computation 39, no. 5 (2005), 523-538.

[3] B. Buchberger, Should Students Learn Integration Rules?, ACM SIGSAM Bulletin 24, no. 1 (January 1990).

[4] A. Cuoco, J. Fey, C. Kieran, L. MacMullin, R. M. Zbiek, Computer Algebra Systems in Secondary School Mathematics Education, National Council of Teachers of Mathematics, 2003.

[5] D. R. Stoutemyer, Crimes and Misdemeanors in the Computer Algebra Trade, Notices of the American Mathematical Society 38, no. 7 (1991), 778-785.

[6] E. Tonisson, Branch Completeness in School Mathematics and in Computer Algebra Systems, submitted, 2007.

[7] E. Tonisson, The Correctness, Completeness and Compactness Standards of Computer Algebra Systems and of School Mathematics, in: Proceedings of the International Symposium Technology and its Integration in Mathematics Education, 2004.

[8] E. Tonisson, (Un)expected infinities in the CAS answers at school, in: Proceedings DES-TIME-2006: Dresden International Symposium on Technology and its Integration into Mathematics Education 2006, bk teachware, 2006.

[9] N. Velikanova, Answers to equations from school textbooks offered by computer algebra systems, Master's thesis, University of Tartu, 2007. 


$$
\text { "tonisson" — 2008/9/1 — 18:16 — page } 138 \text { — \#14 }
$$

138 E. Tonisson, N. Velikanova : Answers offered by CAS to equations from school textbooks

[10] H. Weigand, H. Weller, Changes of Working Styles in a Computer Algebra Environment - the Case of Functions, International Journal of Computers for Mathematical Learning 6, no. 1 (May 2001).

[11] M. J. Wester, A Critique of the Mathematical Abilities of CA Systems, in: Computer Algebra Systems. A Practical Guide, J. Wiley \& Sons, 1999.

[12] M. Zuckerman, Intermediate Algebra, W. W. Norton \& Company, New York, 1976.

ENO TONISSON and NADEZHDA VELIKANOVA

UNIVERSITY OF TARTU

INSTITUTE OF COMPUTER SCIENCE

LIIVI 2

50409 , TARTU

ESTONIA

E-mail: Eno.Tonisson@ut.ee

(Received September, 2007) 